\title{
“¿QUIÉN INUNDA A QUIÉN?” UNA INTERVENCIÓN EN PRIMARIA SOBRE NUESTRA INTERACCIÓN EN EL MEDIO
}

\section{"WHO FLOODS WHO?" AN INTERVENTION IN PRIMARY EDUCATION TO LEARN ABOUT OUR INFLUENCE IN THE ENVIRONMENT}

\author{
Patricia Esteve Guirao \\ Diana González Veracruz \\ Isabel Banos-González
}

\begin{abstract}
Resumen
En este trabajo se presenta el diseño y puesta en práctica de una actividad problema dirigida a implicar al alumnado en la resolución de un conflicto socioambiental cercano: las frecuentes inundaciones de la rambla donde se encuentra su escuela. Mediante el desarrollo de una pequeña investigación, los escolares, organizados en pequeños equipos de trabajo, alcanzaron a comprender la dinámica funcional de las ramblas y fueron capaces de establecer relaciones entre la ocupación territorial de estos cauces y los riesgos de las inundaciones. Además, los escolares lograron consensuar propuestas de soluciones adecuadas, orientadas a reducir estos riesgos mediante la ordenación urbana. Finalmente,
\end{abstract}


en la fase de comunicación, se convocó una reunión con el alcalde de su localidad, a quién entregaron unas cartas elaboradas en el aula que recogían sus propuestas para futuras planificaciones urbanísticas.

\title{
Palabras Clave
}

Educación Primaria, intervención didáctica, problemas socioambientales

\begin{abstract}
This paper presents the design and implementation of a problem activity aimed at involving students in the resolution of a nearby socio-environmental conflict: the frequent flooding of the watercourse, called ramblas, where their school is located. By means of the development of a small inquiry, the students, organised in small working groups, were able to understand the functional dynamics of the watercourses and to establish relationships between the territorial occupation of these ramblas and the risks of flooding. In addition, the students were able to reach an agreement about their proposals for appropriate solutions, aimed at reducing these risks through urban planning. Finally, within the communication phase, a meeting with the mayor of their town was convened, to whom they delivered some letters elaborated in the classroom, which gathered their proposals for future urban planning.
\end{abstract}

\section{Key Words}

Primary Education, didactic intervention, environmental problems

\section{Introducción}

El planteamiento de situaciones de conflicto en el aula debe abordarse desde enfoques globalizadores que promuevan la comprensión de los aspectos ecológicos, sociales, económicos y culturales involucrados. Así, la construcción de este tipo de relaciones, que denominamos socioambientales, puede favorecer que los estudiantes reconozcan nuestro papel en el deterioro del entorno natural y sus consecuencias ambientales y sobre las personas (Marcén y Molina, 2006). 
La perspectiva de este aprendizaje debe superar el conocimiento básico sobre el entorno, hacia una enseñanza donde el desarrollo de este conocimiento se oriente a una educación para el entorno (López y Jiménez Aleixandre, 2001; Forbes y Zint, 2010). Así mismo, será preciso que el alumnado perciba la utilidad de sus acciones y se sienta satisfecho de ellas, haciendo evidente el poder que tenemos como ciudadanos para intervenir en nuestro entorno y su mejora (Tali Tal, 2004).

Sin embargo, actualmente, el alumnado en edad escolar presenta un alto grado de desmotivación, generado en gran medida por concebir la educación como un proceso de acumulación de conceptos abstractos, alejados de los centros de interés de los niños y las niñas (Moreno y Donoso, 2016). Por tanto, uno de los retos es transformar la escuela en nuevos escenarios educativos que permitan a los escolares vincular sus aprendizajes con sus vidas y fomentar la conexión con el medio natural (Borsos, Patocskai y Boric, 2018).

Bajo este objetivo, la selección de las problemáticas a abordar desde el aula será clave, pues la motivación que se despierte en las niñas y los niños por una situación concreta, podrá determinar su nivel de compromiso, además de su capacidad real para actuar en su resolución. De hecho experiencias en este sentido, ponen de manifiesto la capacidad de actuación de los estudiantes de Primaria y el interés que despierta en ellos mismos y fuera de los límites escolares (Esteve y Jaén, 2014).

Así, aproximar al aula la realidad del entorno y promover su interpretación debe representar un objetivo esencial en la etapa de Primaria, aún más en el caso de las ciencias experimentales, que posibilitan en gran medida la comprensión de la realidad del mundo que nos rodea y las transformaciones a las que está sometido. La ciencia en el ámbito escolar no sólo es importante para la comprensión de conceptos de origen científico, sino que también produce un desarrollo del razonamiento necesario para muchas otras facetas de la vida (Souvirón y Méndez, 2015).

La aproximación a los conocimientos científicos resulta fundamental para que los alumnos sean capaces de tomar decisiones y llevar a cabo acciones razonadas, en respuesta a las cuestiones socioambientales. De acuerdo con Campanario (2000), estas orientaciones favorecen que los alumnos tomen un papel activo y comiencen a pensar por sí mismos en la aplicación de la ciencia al contexto cotidiano. Además, con ello se contribuye a fomentar actitudes positivas hacia las disciplinas científicas, pues ayudan a que los estudiantes desarrollen ideas más adecuadas sobre el conocimiento científico como algo cercano y aplicable a la realidad cotidiana.

Sin embargo, hemos de ser conscientes que el alumnado no cambia sus ideas automáticamente al recibir nueva información más ajustada al paradigma científico. Al abordar los problemas socioambientales en las aulas, debemos evitar realizar planteamientos que se reduzcan a la descripción simplificada de fenómenos presentados de forma aislada e independientes de las actividades humanas (Vilches y Gil, 2007). Estos enfoques pueden representar un obstáculo para que los estudiantes comprendan la complejidad de estos problemas y la estrecha vinculación entre la sociedad y los procesos naturales. Esto puede conducir a que sean percibidas como esferas sin relación y, por tanto, puede limitar que los alumnos reconozcan la importancia de actuar como ciudadanos responsables (Freire, 2011).

Por tanto, no solo se trata de conocer el funcionamiento de los ecosistemas y las manifestaciones visibles de las problemáticas de su conservación, sino de alcanzar a 
comprender las dimensiones socioculturales de las causas y sus consecuencias, que son las que explican realmente los conflictos socioambientales de la actualidad (Kramer, 2002). De este modo, además de los conocimientos, cuando se abordan estos conflictos en el aula resulta idóneo fomentar la argumentación y discusión sobre la toma de decisiones entre los alumnos, incluso en edades tempranas (Grace y Byrne, 2010). En la enseñanza sobre estas problemáticas es imprescindible promover el desarrollo de actitudes y comportamientos responsables, donde se tengan en cuenta los intereses de las generaciones futuras (Álvarez Suárez y Vega-Marcote, 2009).

Una de las estrategias didácticas que contribuye a alcanzar estos objetivos y aproximar el proceso de enseñanza a situaciones reales es el aprendizaje basado en el planteamiento y resolución de problemas (Jaén, Esteve y Moreno, 2014). En el contexto de una enseñanza constructivista, el aprendizaje basado en el planteamiento y resolución de problemas sigue como principios que el conflicto cognitivo al abordar una determinada situación estimula el aprendizaje, al activar el conocimiento previo a través de contextos que motiven a los estudiantes. Mediante su análisis y solución, se fomenta su implicación en el problema planteado, el pensamiento crítico y el trabajo colaborativo, dirigidos a alcanzar una toma de decisiones argumentada (Mendoza y Bernabeu, 2006).

Bajo estos principios, en este trabajo se presenta una propuesta didáctica basada en este enfoque, que promueve la concienciación sobre un conflicto del entorno contextual de un centro de Primaria, así como sobre cómo nuestra intervención en el medio conlleva también repercusiones para el ser humano.

\section{Objetivos}

En este trabajo se ha diseñado y puesto en práctica una acción educativa dirigida a implicar a los escolares en la resolución de un problema socioambiental cercano, como es la construcción de su barrio sobre el cauce de una rambla y las frecuentes inundaciones acontecidas. En concreto, se pretende alcanzar los siguientes objetivos específicos:

- Promover una investigación sobre las causas y consecuencias ambientales, sociales y económicas de las inundaciones en su entorno, así como sus posibles soluciones, a partir del establecimiento de relaciones entre las actividades humanas y estos sucesos.

- Evaluar si alcanzan a comprender la dinámica funcional de las ramblas, y reconocer su importancia para adecuar las actividades o proyectos urbanísticos que se realicen en ellas o en sus proximidades.

- Valorar su capacidad para consensuar una actuación orientada a reducir los riesgos de inundación.

\section{Metodología}

\subsection{Contextualización de la experiencia didáctica}

Esta propuesta surge desde el propio proceso de investigación-acción de la tutora de un aula de $4^{\circ}$ de Primaria de un centro de enseñanza público de Molina de Segura (Murcia), que cuenta con 23 alumnos y alumnas. 
Desde su llegada al centro, al inicio de curso, la tutora detectó cierta habituación de sus estudiantes a las inundaciones de la escuela y alrededores, por el desbordamiento de la rambla de El Chorrico, que atraviesa el barrio donde se localiza su centro educativo. Estos fenómenos, relativamente frecuentes en el municipio, representaron una oportunidad para abordar esta problemática en el aula.

La comprensión del funcionamiento de las ramblas es esencial para entender el régimen hidrológico del sureste de la Península lbérica, pues constituyen un rasgo geomorfológico típico de áreas de clima semiárido. Las ramblas se conocen por carecer de caudal hídrico durante largos periodos de tiempo; sin embargo, algunos eventos de lluvias intensas pueden hacer que evacuen grandes cantidades de agua en muy poco tiempo y desborden sus cauces. Por tanto, si la gestión del territorio en su entorno se hace de manera inadecuada, por ejemplo, mediante la ocupación de tipo agrícola o urbana, pueden constituir lugares muy vulnerables frente a las avenidas (Pulido-Bosch, 1993). Su dinámica es, por tanto, diferente a la de los ríos y arroyos, y también sus implicaciones en la planificación territorial.

Lejos del paradigma de ciclo del agua dominante, esta región del sureste español se caracteriza por la presencia de estos cauces no permanentes, cuya existencia es obviada en los libros de texto, como recurso predominante en las aulas de Primaria (Montañés Bayonas y Jaén, 2015). Además, el estudio del paisaje se plantea de modo muy general, sin ningún reflejo de las importantes relaciones e interdependencias existentes con la biosfera, la hidrosfera, la geosfera y la antroposfera.

Todo ello implica que estos manuales presenten un enfoque alejado de situaciones cercanas y reales al alumnado, que dificulta su toma de conciencia sobre la importancia de nuestras actividades y decisiones en la generación de riesgos geológicos como las inundaciones, y las repercusiones que estas tienen sobre nuestras vidas.

En este contexto, se decidió plantear la actividad “¿Quién inunda a quién?”, en el marco de las asignaturas de Ciencias Sociales y Ciencias de la Naturaleza, con un carácter marcadamente interdisciplinar. Esta actividad permite abordar diversos contenidos relacionados con el reconocimiento de la interrelación entre las actividades humanas y el medio ambiente, los cursos de agua y el paisaje, tal y como se recoge en la Tabla 1.

Contenidos que la actividad permite abordar

Observación y descripción de distintos tipos y elementos de un paisaje.

La diversidad geográfica de los paisajes de España: relieve e hidrografía.

La hidrosfera, las masas y cursos de agua.

La intervención humana en el medio. Defensa y mejora del medio ambiente.

Desarrollo de actitudes, individuales y colectivas, frente a determinados problemas medioambientales. 
Tabla 1. Relación de los contenidos más importantes referidos al estudio del paisaje y la interacción con las actividades humanas.

\subsection{Diseño de la propuesta}

Con el fin de superar algunas de las limitaciones identificadas en el apartado anterior y con los objetivos mencionados, se planteó una actividad basada en la estrategia didáctica de resolución de problemas, por su adecuación para favorecer que los escolares alcancen una percepción sistémica de las problemáticas (Caamaño, 2012; Harlen, 2012; Vasconcelos, 2012). Se trata de una estrategia centrada en el alumnado, y basada "en utilizar problemas como punto de partida para la adquisición de nuevos conocimientos" (Vasconcelos, 2012, p. 220); en la que, a partir de un contexto significativo para el alumnado, ellos pueden ir construyendo su propio conocimiento y practicar su transferencia a través de su aplicación dentro de una situación realista. Para ello, la propuesta incluye la formulación de cuestiones clave sobre las causas y consecuencias, el uso de diversas fuentes de datos, el planteamiento o análisis de soluciones y la asunción de compromisos personales para su resolución.

Para la organización de los contenidos, se establecieron diferentes etapas, adaptando el modelo propuesto por Aramburu (2000). Así, se diferenciaron tres grandes fases: i) Planteamiento del problema; ii) Análisis, búsqueda de información y recogida de datos; iii) Definición de posibles soluciones y actuaciones ciudadanas (Tabla 2).

\begin{tabular}{|c|c|c|c|}
\hline Fase & Actividad & Objetivos & $\begin{array}{l}\text { Materiales } \\
\text { necesarios }\end{array}$ \\
\hline $\begin{array}{l}\text { Planteamiento } \\
\text { del problema }\end{array}$ & $\begin{array}{l}\text { Skipper } \\
\text { viene a } \\
\text { Molina }\end{array}$ & $\begin{array}{l}\text { Motivar a los niños y las niñas para el } \\
\text { desarrollo de la actividad. } \\
\text { Plantear el problema e identificar las } \\
\text { cuestiones clave a analizar. } \\
\text { Fomentar la explicitación de las ideas de los } \\
\text { alumnos sobre la rambla. } \\
\text { Orientar la planificación de una pequeña } \\
\text { investigación. }\end{array}$ & $\begin{array}{l}\text { Carta del señor Skipper. } \\
\text { Hoja grupal de } \\
\text { planificación }\end{array}$ \\
\hline $\begin{array}{l}\text { Desarrollo } \\
\text { de la } \\
\text { investigación }\end{array}$ & $\begin{array}{l}\text { El encargo } \\
\text { del señor } \\
\text { Skipper }\end{array}$ & $\begin{array}{l}\text { Promover la confrontación de las ideas de } \\
\text { los alumnos. } \\
\text { Guiar discusiones para poner de manifiesto } \\
\text { la utilidad de la información manejada, para } \\
\text { poder dar respuesta al problema. } \\
\text { Fomentar la adquisición de posiciones } \\
\text { argumentadas sobre el problema: “¿Quién } \\
\text { inunda a quién?" }\end{array}$ & $\begin{array}{l}\text { Hojas de información. } \\
\text { Hoja grupal de informe } \\
\text { final "¿Quién inunda a } \\
\text { quién?". }\end{array}$ \\
\hline $\begin{array}{l}\text { Búsqueda de } \\
\text { soluciones }\end{array}$ & $\begin{array}{l}\text { El juego } \\
\text { del } \\
\text { Molinapoly }\end{array}$ & $\begin{array}{l}\text { Mantener el compromiso y la participación } \\
\text { en la fase de búsqueda de soluciones. } \\
\text { Proponer una situación nueva para que los } \\
\text { alumnos apliquen los aprendizajes } \\
\text { desarrollados. }\end{array}$ & $\begin{array}{l}\text { Tablero con la imagen } \\
\text { de los } \quad \text { terrenos } \\
\text { urbanizables r del } \\
\text { municipio. }\end{array}$ \\
\hline
\end{tabular}




\section{Promover la realización de propuestas de Figuras para llevar a soluciones argumentadas. ordenación. \\ Carta individual a las autoridades.}

Tabla 2. Relación de actividades, objetivos y materiales que conforman la propuesta.

\section{Planteamiento del problema: "Skipper viene a Molina"}

El problema se plantea mediante una carta enviada por un personaje de ficción muy conocido por los escolares, en la que les cuenta que está planeando construirse una casa junto a la rambla de El Chorrico, y les pide que le informen sobre algunos aspectos que tienen que ver con dicha rambla (Figura 1).

Los escolares, organizados en pequeños equipos de trabajo, deben consensuar cuál de estos aspectos investigar y, en coherencia, planifican una pequeña investigación, que es recogida en la hoja grupal de planificación.

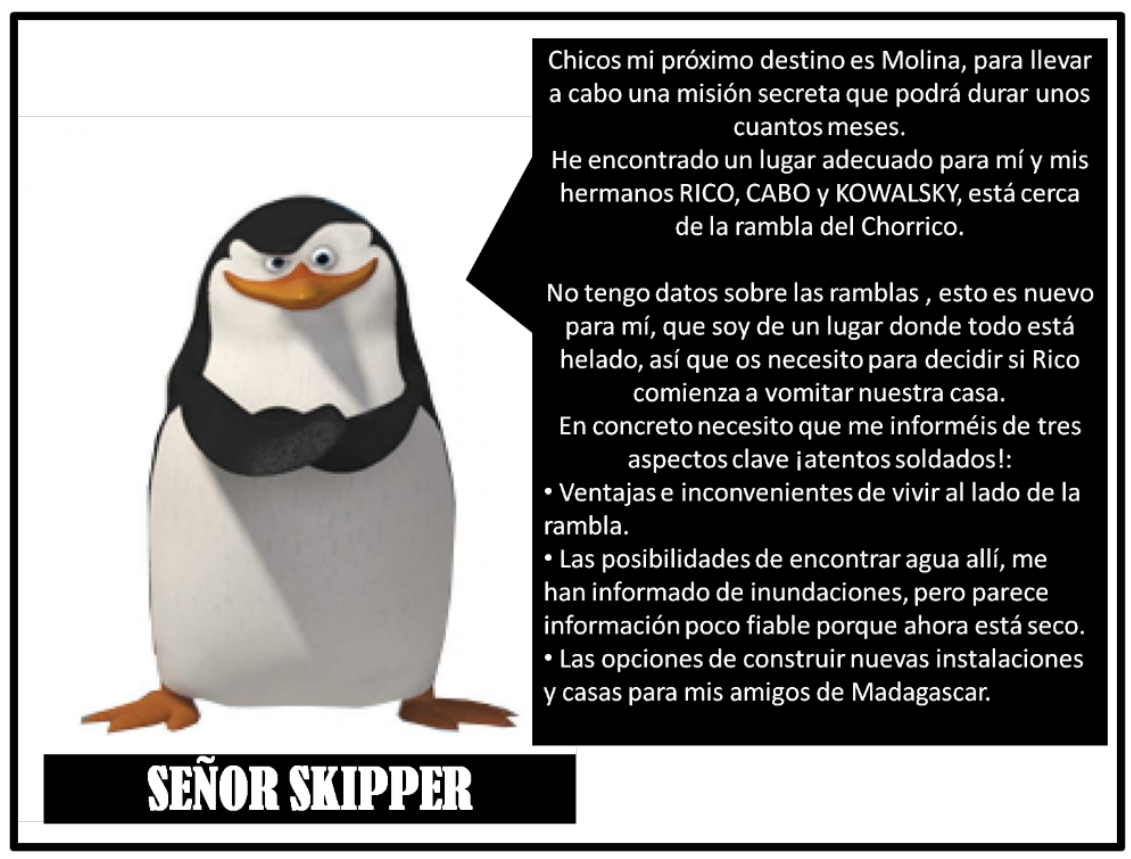

Figura 1. Planteamiento del problema.

\section{Desarrollo de la investigación: "El encargo del señor Skipper”}

Para esta fase, se preparan una serie de hojas de información (Figura 2) para que los niños accedan a diversos datos relevantes, que incluyen:

- La dinámica general de las ramblas, particularizada en la de El Chorrico: régimen de presencia de agua, caudales, velocidad y otras características. 
- Efectos de la crecida de las ramblas y, en concreto, sobre su barrio y otras zonas del municipio; así como los costes económicos de tales efectos.

- Imagen aérea en la que se aprecia la distribución de los barrios en Molina respecto a la rambla en el momento en el que se realizó la actividad, año 2014; e imagen de la misma zona en 1945.

- Propuestas técnicas y normas existentes para evitar los efectos de las crecidas de las ramblas.

En función de la planificación realizada por cada equipo de trabajo, se les facilita las diferentes hojas de información asociadas. Al final de cada una de ellas, aparecen unos interrogantes que conducen al siguiente paso de la investigación, orientando el proceso. De este modo, se pretende facilitar la autonomía del alumnado, respetando distintos ritmos e intereses, y que el papel de la docente se centre en la resolución de posibles dudas. Los resultados de la investigación, quedan recogidos en el informe final: "¿Quién inunda a quién?".
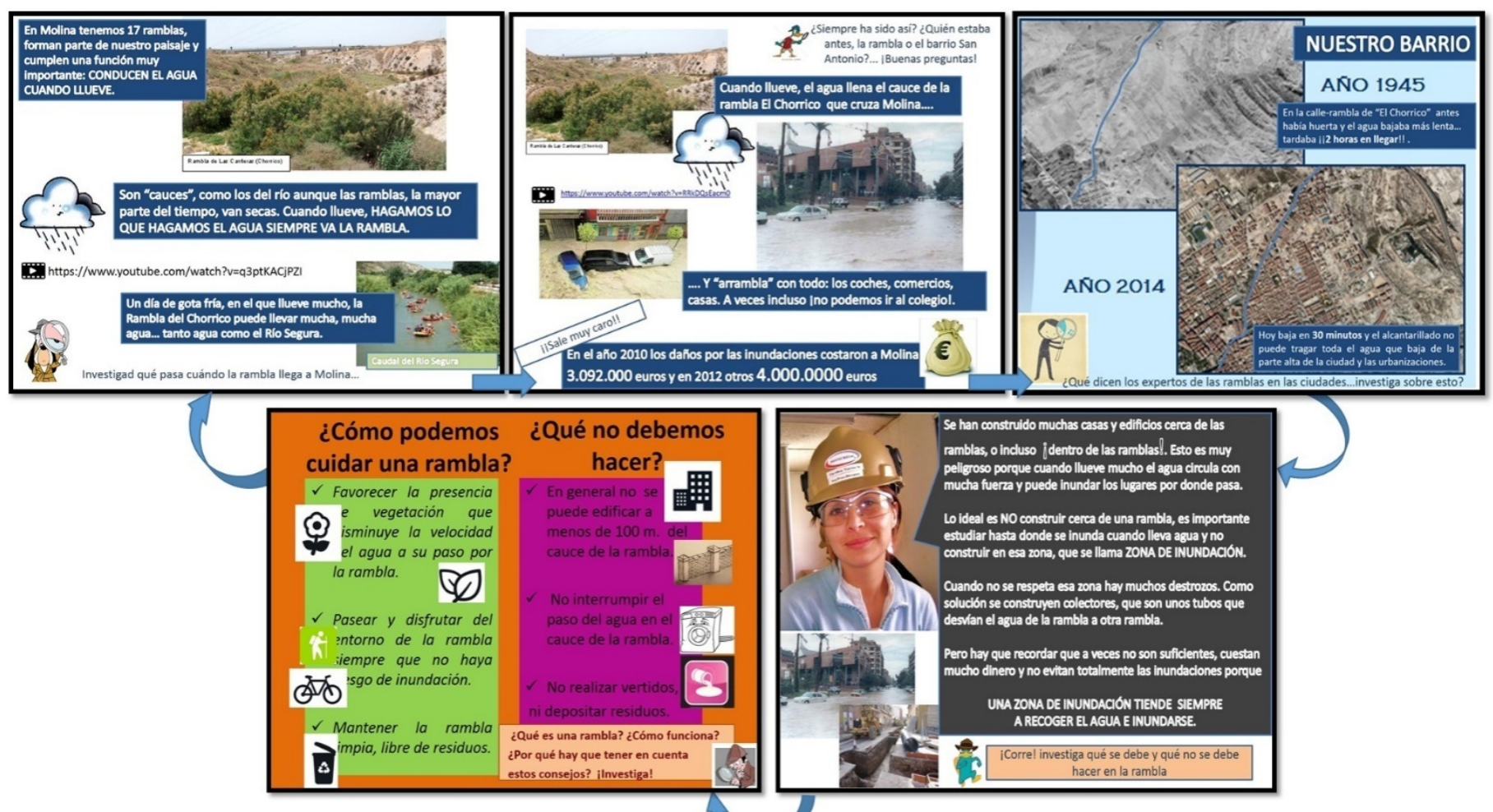

Figura 2. Hojas de información para que el alumnado desarrolle la investigación. Desde arriba a la izquierda: a) Dinámica general de las ramblas; b) Efectos de las crecidas de las ramblas; c) Distribución de los barrios en Molina respecto a la rambla, en 2014 y 1945; d y e) Propuestas técnicas y normas.

\section{Búsqueda de soluciones: "Molinapoly"}


Esta fase se orienta a aplicar los aprendizajes alcanzados en una nueva situación. Para ello, cada equipo dispone de un tablero con la imagen de unos terrenos urbanizables de su municipio, atravesados por varias ramblas, donde el personaje inicial y sus amigos podrían construir sus viviendas. Así mismo, se reparten pegatinas y figuras que simulan árboles, casas y servicios públicos, de modo que cada equipo pueda configurar un proyecto de barrio.

El objetivo es que cada equipo de trabajo tome decisiones para organizar los distintos elementos, de acuerdo con los riesgos asociados a la existencia de las ramblas. Tras una primera discusión, se ofrecen pequeñas pautas sobre las dimensiones de la zona de inundación, la importancia de la existencia de vegetación en el cauce, distancia mínima de la rambla a la que deben situar determinados servicios, etc. Para realizar estas mediciones, se incluye una escala sencilla que pueden manejar utilizando sus reglas. Al finalizar esta fase, cada grupo expone su propuesta de barrio al resto de los compañeros, justificando sus decisiones.

Finalmente, se propone redactar una carta dirigida al servicio competente en urbanismo del Ayuntamiento de su localidad, en la que explican los resultados de su investigación, sus propuestas para planificar los terrenos urbanizables y otras medidas para evitar inundaciones.

\subsection{Instrumentos para el análisis de los resultados}

Para la evaluación de la consecución de los objetivos propuestos en la actividad, mayoritariamente cualitativa, se han utilizado los siguientes instrumentos:

- Rúbrica de observación directa, con la que se pretende valorar la dinámica de trabajo de cada uno de los equipos de trabajo (Tabla 3 ).

- Grabación en vídeo y audio de los escolares para recoger sus comentarios mientras realizan la tarea y detectar posibles dificultades. Las grabaciones de audio fueron transcritas y ordenadas según las fases de la actividad.

- Diseño de los tableros del Molinapoly, para evaluar si el alumnado consigue aplicar los aprendizajes alcanzados en una nueva situación.

- Cartas individuales dirigidas al Alcalde de Molina de Segura. El análisis de sus escritos se realizó siguiendo el método inductivo, mediante codificación y categorización en 4 categorías. Para asegurar la fiabilidad del proceso, participaron dos investigadores independientes, con un coeficiente de acuerdo de 0,85 , que se considera adecuado (Miles y Huberman, 1994).

\begin{tabular}{|l|l|}
\hline \multicolumn{1}{|c|}{ Fase } & \multicolumn{1}{c|}{ Indicadores de evaluación } \\
\hline \multirow{3}{*}{$\begin{array}{l}\text { Planteamiento } \\
\text { del problema }\end{array}$} & Expresan ideas o experiencias previas relacionadas con la situación planteada. \\
\cline { 2 - 2 } & Reconocen algunas ventajas y desventajas de edificar cerca de las ramblas. \\
\cline { 2 - 2 } & Se muestran activos e interesados por el tema. \\
\cline { 2 - 2 } & Respetan las ideas o posicionamientos de otros compañeros. \\
\hline & Leen la información y reconocen de forma clara lo que hay que hacer. \\
\hline
\end{tabular}




\begin{tabular}{|c|c|}
\hline \multirow{4}{*}{$\begin{array}{l}\text { Fase de } \\
\text { investigación }\end{array}$} & Discuten entre ellos sobre la información. \\
\hline & Se centran en aspecto relevantes. \\
\hline & Se muestran interesados en el tema, y solicitan información adicional. \\
\hline & Alcanzan posiciones argumentadas sobre “¿Quién inunda a quién?” \\
\hline \multirow{6}{*}{$\begin{array}{l}\begin{array}{l}\text { Búsqueda de } \\
\text { soluciones }\end{array}\end{array}$} & $\begin{array}{l}\text { No necesitan instrucciones del Molinapoly para considerar algunas de las limitaciones } \\
\text { aprendidas en la fase anterior. }\end{array}$ \\
\hline & La visión espacial es adecuada y les permite interpretar los elementos en los mapas. \\
\hline & $\begin{array}{l}\text { Disponen adecuadamente los elementos de la urbanización en función de la existencia } \\
\text { de ramblas. }\end{array}$ \\
\hline & Proponen soluciones argumentadas. \\
\hline & Se muestran involucrados en la ordenación del territorio. \\
\hline & $\begin{array}{l}\text { Son capaces de plasmar en sus cartas los contenidos trabajados, } r \\
\text { causas y consecuencias; } y \text {, además, plantean soluciones adecuadas. }\end{array}$ \\
\hline
\end{tabular}

Tabla 3. Rúbrica para la evaluación de la actividad.

\section{Resultados y discusión}

El análisis y discusión de los resultados se estructura siguiendo las tres fases de la actividad y los objetivos planteados en el presente trabajo.

En el planteamiento del problema, cuando los escolares expresaron sus ideas iniciales, se observó una amplia familiarización con el término de rambla. Sin embargo, sus comentarios apuntaban a que estos cauces parecían ser percibidos, por la gran mayoría, como un elemento del paisaje urbano. En concreto, la identificaban como una calle más del barrio, pasando casi invertida su función como cauce natural de las aguas.

Todos han vivenciado algún fenómeno de inundación en la zona, recordando con detalle algunas anécdotas de destrozos, pero sin llegar a plantearse por qué ocurre esto ni qué se podría hacer para evitarlo. A continuación, se recogen algunas de sus intervenciones, extraídas de las transcripciones de los audios:

Alumno 1: "Aquel día llovía tan fuerte y bajaba el agua así, ifiuuum!. Los contenedores le pegaron topazos a los coches, hasta que se quedó parado contra una furgoneta".

Alumna 2: "En la casa de mi madrina entró el agua y se le mojaron todos los muebles de barro".

Sin embargo, cuando se les invitó a discutir sobre las desventajas de que el personaje construya su casa en una zona atravesada por ramblas, ninguno llegó a mencionar los riesgos por inundación, sino que se centraron en otros aspectos. Por ejemplo, el alumno 3 decía: "El inconveniente más grande es que en la rambla no hay hielo, y los pingüinos se van a asar de calor".

En general, en esta primera parte, todos se muestraron participativos, respetando los turnos de palabra; y, dentro de cada equipo, llegaron fácilmente a consensos sobre qué aspecto empezar a investigar. 
En la fase de desarrollo de la investigación, en un primer momento, todos miraban las imágenes y comentaban situaciones familiares relacionadas. Es el caso de la alumna 4: "Mira, aquí me deja mi padre con el coche para venir a clase" (En referencia a la ficha de trabajo de la figura $2 b$ ).

Aunque uno de los grupos requirió de la intervención de la docente para iniciar la investigación y seleccionar información de relevancia, el resto de equipos trabajaron con bastante autonomía. En general, la dinámica de trabajo observada consistía en que uno de los miembros en cada equipo leía en voz alta la información de la ficha y después hablaban entre ellos, centrando los comentarios en aspectos útiles para completar el informe final. Los diálogos entre ellos denotaron un alto grado de implicación en la misma, sin necesidad de la intervención de la maestra:

Alumna 5 (encargada de leer la hoja de información): “¿Quién estaba primero, la rambla o el barrio de San Antonio?".

Alumno 6: "Pues el barrio".

Alumna 7: "Yo creo que no, que primero la rambla, y por eso el barrio tiene ese nombre (...). Que lo conocen como el barrio de El Chorrico".
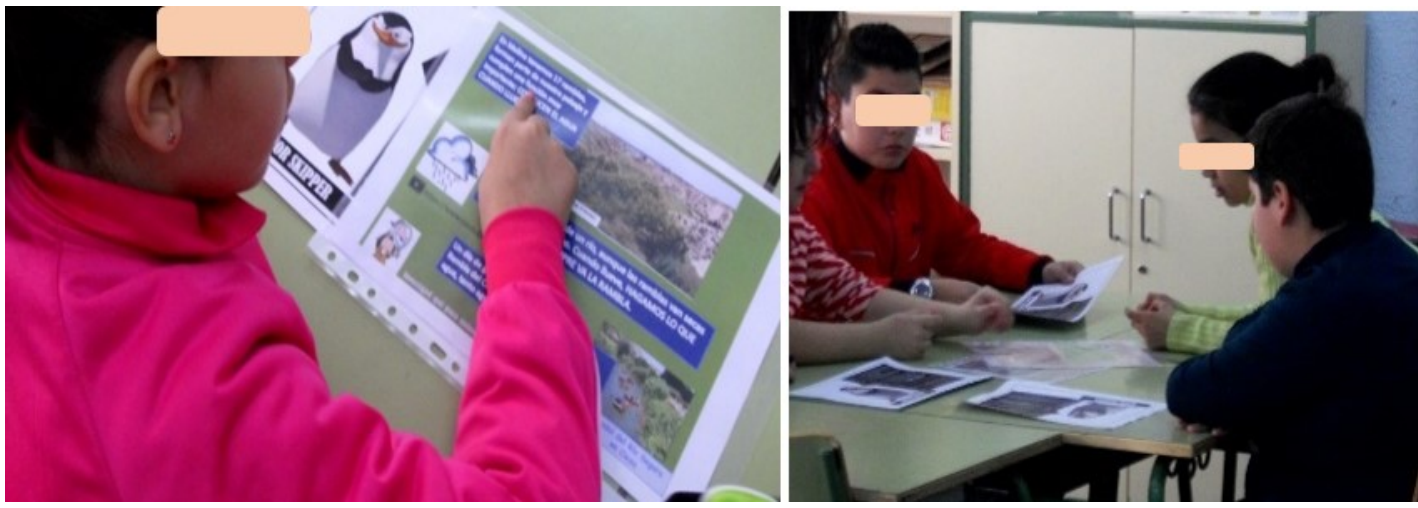

Figura 3. Algunos momentos del desarrollo de la fase de investigación.

Por otro lado, la ficha de información que más dificultades planteaba para los escolares fue la que recoge imágenes de la zona, tanto en 1945 como en 2014. Parece que les resultaba complejo interpretar que su barrio, en el pasado, era una zona de huerta, con una configuración completamente distinta a la actual. En la discusión de aula, tampoco resultaba sencillo que reconocieran cómo los cambios en el paisaje y, concretamente, en los usos del suelo (de huerta a suelo urbanizado), afectan a la velocidad de la corriente de agua y, por tanto, al riesgo de inundación. Por lo tanto, la ficha $2 \mathrm{c}$ requirió de la intervención de la maestra para interpretar el mapa y las consecuencias de los cambios de usos de suelo.

A pesar de estas dificultades, en su informe “¿Quién inunda a quién?”, todos los grupos reconocieron que el barrio se había construido sobre la rambla, y que ese hecho favorece que se produzcan las inundaciones. Por ejemplo, en el informe del grupo 3 lo expresaban de la siguiente manera: "es como si hubiesen construido el barrio dentro de un río, pero que casi todo el tiempo está seco". 
En la última fase de búsqueda de soluciones, al enfrentarse al reto del juego de "Molinapoly", se constató que en todos y cada uno de los grupos, alguno de los miembros coloca los símbolos de la vegetación y de los edificios sobre el tablero sin considerar criterios adecuados relacionados con el riesgo de inundación. Sin embargo, destaca que, en cuatro de los cinco grupos, se produjo un proceso de autoregulación en las decisiones por parte de los propios miembros del equipo mediante la discusión, como muestra la siguiente conversación:

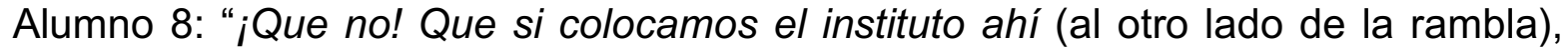
cuando llueva no van a poder pasar".

Alumno 9: "Pues mejor, así no van a clase, y pierden el día".

Alumno 8: "Pero si les pilla la lluvia en el instituto, ¿qué?... Se quedan allí dos días sin poder volver".

Sólo uno de los grupos necesitó la mediación de la docente para ayudarlos a tomar decisiones al respecto.

El análisis de los cinco tableros elaborados por cada uno de los grupos y su posterior puesta en común, mostró que realizaban propuestas bien diferenciadas, y todas ellas ajustadas a los criterios de seguridad para limitar los riesgos de inundación asociados a las ramblas. En este sentido, aunque ya reconocían la necesidad de alejar las viviendas y otros elementos de las ramblas, conocer y disponer de pautas para ubicar los elementos del barrio, ofreció a los escolares la oportunidad de delimitar de forma específica la zona de inundación y dar prioridad a la vegetación en los márgenes del cauce (Figura 4). En este sentido, una de las alumnas daba indicaciones a su compañero de equipo de la siguiente manera:

Alumna 10: "No pongas más árboles ahí (un jardín, en el centro del casco urbano), que los árboles también los necesitamos cerca de la rambla".

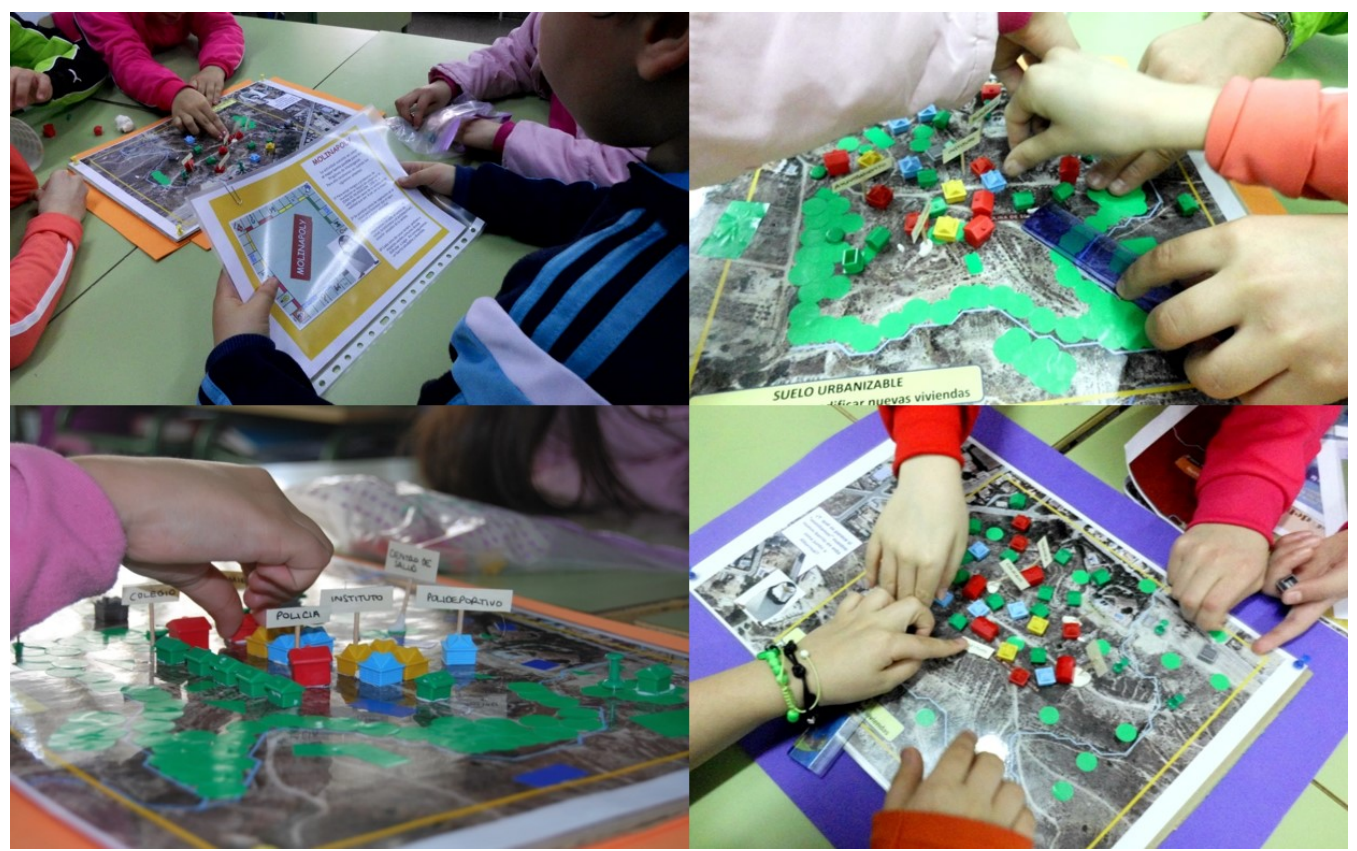

Figura 4. Algunos momentos del desarrollo del Molinapoly, en la fase de búsqueda de soluciones. 
Finalmente, el análisis de las cartas realizadas por las niñas y los niños permite establecer cuatro categorías en relación al nivel de complejidad argumental que expresan en sus escritos (Tabla 4). De este modo, se observa que en torno a una quinta parte del alumnado, no reflejó en sus cartas la responsabilidad humana en el riesgo de inundación cuando se construye en las proximidades de las ramblas. Esta categoría 1 recoge las producciones de aquellos alumnos que sólo se centraron en consecuencias y, concretamente, en hechos catastróficos. Por ejemplo, el alumno 11: "En Molina, cuando llueve fuerte se forman inundaciones, y el agua se lo va a lleva todo".

\begin{tabular}{|llc|}
\hline Categorías & Descripción & Frecuencia \\
\hline Categoría 1 & $\begin{array}{l}\text { Sólo menciona consecuencias, y se centra en hechos llamativos o } \\
\text { catastróficos }\end{array}$ & 5 \\
\hline Categoría 2 & $\begin{array}{l}\text { Reconoce la interacción humana sobre el medio mediante la } \\
\text { construcción de relaciones causa-efecto }\end{array}$ & 12 \\
\hline Categoría 3 & $\begin{array}{l}\text { Conecta causas y consecuencias de forma argumentada y, además, } \\
\text { ofrece soluciones preventivas válidas }\end{array}$ & 4 \\
\hline Categoría 4 & $\begin{array}{l}\text { Además de lo anterior, ofrece otro tipo de soluciones válidas } \\
\text { destinadas a minimizar el riesgo ya existente. Además, reconoce la } \\
\text { necesidad de enseñar a todos los vecinos lo que han aprendido }\end{array}$ & 2 \\
\hline
\end{tabular}

Tabla 4. Frecuencia de las categorías y descripción de las mismas.

La mayoría del alumnado alcanzó la categoría 2, en la que se relaciona algunas decisiones humanas con la ocurrencia de inundaciones, como se demuestra en el ejemplo de la alumna 12: "Como cuando llueve la rambla se llena de agua; al haber colocado el colegio en el cauce, entrará el agua también”.

Del resto, cuatro alumnos fueron capaces de avanzar en la argumentación sobre las causas y consecuencias, y ofrecer soluciones válidas centradas en criterios de ordenación del territorio. Este es el caso de la carta del alumno 13, que señala: "Hemos aprendido que para que no se inunden las nuevas urbanizaciones y colegios no se pueden construir sobre el cauce de la rambla. Hay que dejar 100 metros y cuanto más lejos mejor".

Finalmente, otras 2 alumnas llegaron a alcanzar la categoría 4, puesto que sus propuestas de soluciones son más precisas y se orientan, no sólo a prevenir, sino a minimizar el riesgo ya existente. Además, en sus cartas reconocen la necesidad de formar a la ciudadanía sobre los riesgos que entraña la urbanización en el cauce de las ramblas:

Alumna 14: "También, hemos visto que poniendo vegetación cerca del cauce y colectores, se puede bajar el daño para las personas. (...). Tenemos que contarle a todos los vecinos qué pasa si ponen sus casas y sus tiendas en los cauces, porque las van a perder". 
Como se ha mencionado anteriormente, las cartas fueron enviadas al alcalde del municipio, quién, posteriormente, invitó a este grupo de alumnos y alumnas a asistir al Ayuntamiento para dialogar sobre lo que habían aprendido.

Durante la reunión en el consistorio, algunos niños y niñas fueron más participativos que otros; en general, presentaron ciertas dificultades para mostrar su análisis crítico sobre la ordenación urbanística del municipio, con reticencias para expresar frente al alcalde y algunos asistentes aspectos que habían reconocido en sus cartas como poco adecuados sobre dicha ordenación. No obstante, poco a poco se mostraron más relajados y participativos en general; $\mathrm{y}$, apoyándose en las fotografías ampliadas de sus tableros de Molinapoly, lograron explicar al conjunto de asistentes la importancia de planificar futuras construcciones urbanísticas del municipio para evitar inundaciones, considerando la dinámica de las ramblas.

Posteriormente y ya en el aula, se discutió sobre esta actuación, donde los estudiantes reconocieron la importancia de que la ciudanía se implique y lleve los resultados de sus análisis críticos de la información a los responsables políticos, acompañándolos de propuestas de mejora.

\section{Conclusiones}

A pesar de que las frecuentes inundaciones en la rambla de su municipio son familiares para los niños y niñas de esta clase de $4^{\circ}$ de Primaria, al inicio de la actividad no se mostraron capaces de identificar sus causas, más allá de ligarlas a fenómenos meteorológicos de lluvias intensas. La rambla era percibida como un elemento más del paisaje urbano en el que viven, pasando casi inadvertida como cauce natural de las aguas.

Los resultados descritos sugieren que una propuesta de intervención como la que se presenta en este trabajo, en la que, a partir de una situación problemática cercana al alumnado, los participantes debían investigar sobre las causas y consecuencias ambientales, sociales y económicas relacionadas con el problema, ha favorecido su implicación en el proceso de aprendizaje, así como la comprensión de los contenidos trabajados. De este modo, se puede concluir que la mayoría del alumnado ha alcanzado a comprender la dinámica funcional de las ramblas. Así mismo, parecen reconocer cómo la intervención humana influye en el riesgo de inundación $y$, por tanto, la importancia de adecuar las actividades o proyectos que se realicen en sus proximidades (Vasconcelos, 2012).

Respecto al trabajo grupal requerido para el diseño de los tableros del Molinapoly, se valora positivamente la capacidad de los escolares para consensuar actuaciones sobre el territorio orientadas a reducir los riesgos de inundación. Todos los grupos, excepto uno, fueron capaces de llegar a acuerdos al respecto, sin la intervención de la docente.

Por otro lado, la evaluación de las cartas elaboradas por los niños y niñas ha mostrado la capacidad de la amplia mayoría para reconocer, ahora ya de manera individual, las relaciones entre ciertas actividades humanas y las inundaciones de la rambla, mediante la construcción de relaciones causa-efecto. Destaca el caso de dos de las cartas, en las que, además, se subrayaba la necesidad de informar a la ciudadanía sobre los riesgos que podrían suponer dichas actividades, si no se hace una planificación del territorio adecuada.

Por tanto, estos resultados respaldan lo sugerido por Harlen (2012), sobre la necesidad de potenciar el desarrollo de estrategias de enseñanza que favorezcan la 
indagación, en las que puedan identificar un problema concreto y cercano, trabajar de manera interdisciplinar distintas fuentes de información y proponer soluciones al respecto. De acuerdo con Campanario (2000), es esencial que los escolares reconozcan la utilidad de sus aprendizajes, así como de las soluciones que alcanzan a proponer. Así se puso de manifiesto tras la reunión con el alcalde del municipio, al exponer sus propuestas para futuras planificaciones urbanísticas, respetando los cauces de las ramblas y haciendo los barrios de su entorno más habitables.

Somos conscientes de que la escasa replicabilidad de esta experiencia centrada en un contexto determinado, así como de los materiales elaborados especialmente para el desarrollo de este caso, podría ser una limitación para otros colegas en contextos geográficos distintos. Sin embargo, consideramos esencial generar dinámicas como esta en el aula, en las que el alumnado se sienta realmente familiarizado con el contexto de los contenidos que se abordan, y reconozcan la utilidad de los aprendizajes y de las decisiones que consensuan.

\section{Bibliografía}

Agudo, M.T., Cano, M.J. \& Burgos, C. (2016). ¡Disfruta y aprende!: la alegría de aprender a través de tareas integradas descubriendo nuevas emociones, sentimientos y la realidad que nos rodea. Aula de Encuentro, 18, 2, 32-55.

Álvarez Suárez, P. \& Vega-Marcote, P. (2009). Actitudes ambientales y conductas sostenibles. Implicaciones para la educación ambiental. Revista de psicodidáctica, 14, 2, 245-260.

Aramburu, F. (2000). Medio ambiente y Educación. Madrid, España: Síntesis de Educación.

Borsos, E., Patocskai, M. \& Boric, E. (2018). Teaching in nature? Naturally!. Journal of Biological Education, 1-11. (DOI: https://doi.org/10.1080/00219266.2017.1420679)

Caamaño, A. (2012). ¿Cómo introducir la indagación en el aula? Alambique: Didáctica de las ciencias experimentales, (70), 83-92.

Campanario, J.M. (2000). El desarrollo de la metacognición en el aprendizaje de las ciencias: estrategias para el profesor y actividades orientadas al alumno. Enseñanza de las Ciencias, 18, 3, 369-380.

Esteve, P., \& Jaén, M. (2014). Cómo indagan los niños de Primaria sobre el problema de las medusas en el Mar Menor y qué medidas proponen. Aula de Innovación Educativa, 238, 46-49.

Forbes, C.T. \& Zint, M. (2010). Elementary teachers' beliefs about, perceived competencies for, and reported use of scientific inquiry to promote student learning about and for the 
environment. The Journal of environmental education, 42, 1, 30-42. (DOI: https://doi.org/10.1080/00958961003674673)

Freire, H. (2011). Educar en verde. Ideas para acercar a niños y niñas a la naturaleza. Barcelona, España: GRAÓ.

Grace, M. \& Byrne, J. (2010). Engaging pupils in decision-making about biodiversity conservation issues. School Science Review, 91(336), 73-80.

Harlen, W. (2012). Inquiry in science education. En: S. Borda Carulla (coord.), Resources for implementing inquiry in science and mathematics at school. Recuperado de http://www.fibonacci-project.eu.

Jaén, M., Esteve, P. \& Moreno, P.P. (2014). Indagar sobre la pérdida de biodiversidad desde el consumo alimentario ciudadano. Alambique: Didáctica de las ciencias experimentales, $78,43-50$.

Kramer, F. (2002). Manual práctico de educación ambiental. Técnicas de simulación, juegos y otros métodos educativos. Madrid, España: Catarata.

López, R. \& Jiménez Aleixandre, M.P. (2001). Qué tipo de educación ambiental concibe y ejecuta el profesorado. ¿Se hace la misma qué se piensa? Adaxe, 17, 287-309.

Marcén, A.C. \& Molina, P.J. (2006). La persistencia de las opiniones de los escolares sobre el medio ambiente. Una particular visión retrospectiva desde 1980 a 2005. Valsaín: CNEAM.(http://www.mapama.gob.es/es/ceneam/articulos-deopinion/2007 01marcen tcm30-163503.pdf)

Mendoza, X. \& Bernabeu, M.D. (2006). Aprendizaje basado en problemas. Innovación Educativa, 6, 35, 1-12.

Miles, M. \& Huberman, A. (1994). Qualitative data analysis. 2nd ed. Thousand Oaks, CA: Sage.

Montañés Bayonas, S. \& Jaén, M. (2015). ¿Qué características presentan los contenidos relacionados con las problemáticas ambientales propuestos en los libros de texto de $3^{\circ}$ de la eso? Revista Eureka sobre Enseñanza y Divulgación de las Ciencias, 12, 1, 130-148.

Moreno, M. \& Donoso, M. (2016). ECOESCUELA: una estrategia para la evaluación de competencias en el alumnado de Educación Superior. Aula de Encuentro, 18, 2, 5675.

Pulido-Bosch, A. (1993). Las ramblas mediterráneas; condicionantes geomorfológicos e hidrológicos. In IEA. Las ramblas mediterráneas, Actas de la VI Aula de Ecología. (pp. 131-140). Madrid: Instituto de Estudios Almerienses. 
Souvirón, P. \& Méndez, D. (2015). Desarrollo del pensamiento lógico por medio de la metodología de enseñanza ISLE. Aula de Encuentro, 17, 1, 212-238.

Tali Tal, R. (2004). Community-based environmental education-a case study of teacherparent collaboration. Environmental Education Research, 10(4), 523-543. (DOI: https://doi.org/10.1080/1350462042000291047)

Vasconcelos, C. (2012). Teaching environmental education through PBL: Evaluation of a teaching intervention program. Research in Science Education, 42(2), 219-232. (DOI: https://doi.org/10.1007/s11165-010-9192-3)

Vilches, A. \& Gil, D. (2007). Emergencia planetaria: necesidad de un planteamiento global. Educatio Siglo XXI, 25, 19-49.

\section{REFERENCIA BIBLIOGRÁFICA}

Esteve, P, González, D y Banos- González, I. (2019). “¿quién inunda a quién?” una intervención en primaria sobre nuestra interacción en el medio. Aula de Encuentro, volumen 22 (número 1), pp. 105-122.

Patricia Esteve es

Profesora del Dpto. de Didáctica de las Ciencias Experimentales de la Universidad Murcia Correo-e: p.esteve@um.es

Diana González Veracruz es Profesora del CEIP San Antonio (Molina de Segura, Murcia) Correo-e: diana.gonzalezveracruz@gmail.com

Isabel Banos-González es Profesora del Dpto. de Didáctica de las Ciencias Experimentales de la Universidad Murcia Correo-e: ibbg1@um.es 
Esteve, P, González, D y Banos- González, I. (2019). “¿quién inunda a quién?” una intervención en primaria sobre nuestra interacción en el medio. Aula de Encuentro, volumen 21 (1), pp. 105-122. 